\title{
The pharmacist in retrospect
}

\section{Per Hartvig}

"The pharmacist lost his profession when he could not feel the taste of his drug, not smell it, could not recognise its structure, could not extract and purify it, did not know how to formulate it, did not know how to store it and how to describe it. He has then searched the profession ever since. He has not found it". Yngve Torud at the 1st EUFEPS Conference, 1993

This change might have happened in the mid-1950s. Is it that bad? The skills of the old pharmacist are lost with the generation now dying. This is really sad and there is also a strong need to keep the knowledge for coming generations to highlight the great skills and competences that were linked to the profession.

However, new niches have emerged which have pharmacists as initiators and important fashion leaders. One was 'good manufacturing practice' that came up at the same time and moved the quality of medicines and processes for their manufacture forward. This forefront position has been maintained by pharmacists ever since. Other aspects of quality and in different members of the GXP family also generated interest and support from

Correspondence to Per Hartvig, Editor, EJHP Practice \& Science, Department of Pharmacology and Pharmacotherapy, SUND, University of Copenhagen, Universitetsparke 2, Copenhagen 2100, Denmark; peh@farma.ku.dk pharmacists, although sometimes beyond common sense. The characteristics of a pharmacist (highly quality minded, safety thinking, precise, controlling and structured), which have been shown in scientific studies, played an important role in their impact. GMP has even been translated as 'Good made pharmacists'.

Another area was pharmacokinetics. Pharmacists have the skills and knowledge for bioanalysis, calculations and statistics, which are the mainstays of kinetics. Although pharmacokinetics might be regarded as a support science, it has been developed tremendously under the auspices of the pharmacists into knowledge on the relation of pharmacokinetics to pharmacodynamics using advanced simulations and modelling to understand and explain complex interactions. The science has developed into related areas, such as clinical trials and clinical mechanistic studies, as well as the area of developing optimal research designs. The statistical calculations and models are well developed and most pharmacists have a working knowledge of how to calculate basic parameters such as volume of distribution, clearance and bioavailability. However, they often have difficulties understanding and using the information in terms of reasoning of mechanisms of action, making changes when conditions are altered and providing dosing advice when all of the information is not available on drug disposition in the single patient.
There are other areas which have benefitted from the initiatives and interests of pharmacists and where they have contributed to development and obtained a leading position. These are: pharmaco-epidemiology, although this area is still limited with respect to predicting outcome; drug formulation and drug stability aspects on a small scale; patient/ medication safety; clinical pharmacy, which has to show value for money; and clinical trial methodology and pharmacovigiliance, areas where we are still searching for good methods for early warning.

Times have changed. The profession has broadened and it is still not possible to describe the profession in a few words. The new fields are all present within the hospital pharmacy. There is a great need to show the steps forward that have been made in areas where pharmacists are playing a pronounced role and even leading development. The best way to document development is publishing, and in a peer reviewed journal which covers all aspects of pharmacy science, such as the European Journal of Hospital Pharmacy, now being published by a new publisher.

\section{Competing interests None.}

Provenance and peer review Commissioned; not externally peer reviewed.

Accepted 22 January 2012

European Journal of Hospital Pharmacy 2012;19:2. doi:10.1136/ejhpharm-2012-000067 